\title{
Effect of Mirogabalin on Chemotherapy-induced Peripheral Neuropathy Caused by Gemcitabine Plus Nab-paclitaxel Therapy in Pancreatic Cancer Patients: A Pilot Study
}

\author{
Yusuke Takasaki \\ University of Juntendo \\ Toshio Fujisawa \\ University of Juntendo \\ Mako Ushio \\ University of Juntendo \\ Sho Takahashi \\ University of Juntendo \\ Wataru Yamagata \\ University of Juntendo \\ Akinori Suzuki \\ University of Juntendo \\ Koichi Ito \\ University of Juntendo \\ Kazushige Ochiai \\ University of Juntendo \\ Ko Tomishima \\ University of Juntendo \\ Shigeto Ishi \\ University of Juntendo \\ Hiroyuki Isayama ( $\nabla$ h-isayama@juntendo.ac.jp ) \\ University of Juntendo
}

\section{Research Article}

Keywords: Mirogabalin, CIPN, GnP

Posted Date: November 8th, 2021

DOI: https://doi.org/10.21203/rs.3.rs-948459/v2 
License: (c) (i) This work is licensed under a Creative Commons Attribution 4.0 International License. Read Full License 


\section{Abstract}

Purpose

Gemcitabine/nab-paclitaxel therapy $(\mathrm{GnP})$ is widely used to treat pancreatic cancer $(\mathrm{PC})$, but chemotherapy-induced peripheral neuropathy (CIPN) is common. Mirogabalin is a novel drug for treating peripheral neuropathy. We investigated the effects of mirogabalin on CIPN due to GnP.

Methods

Patients who had received GnP for PC and had taken mirogabalin for CIPN, were included. Patients completed a questionnaire about their symptoms before and after taking mirogabalin. The outcome was the change in numbness and tingling scores on the patient-reported outcomes version of the Common Terminology Criteria for Adverse Events, the European Organization for Research and Treatment of Cancer Quality of Life Questionnaire-Chemotherapy-Induced Peripheral Neuropathy (EORTC-QLQCIPN20), numerical rating scale, and adverse events (AEs).

Results

Increased numbness and tingling severity (1.84 vs. $1.76 ; P=0.63)$ and interference $(1.42$ vs. $1.44 ; P=0.80)$ were not seen in any of the 25 enrolled patients. The scores on the sensory subscale of the EORTC-QLQCIPN improved significantly after treatment (17.5 vs. $15.7 ; P=0.02)$. AEs occurred in 22 patients (88\%), but there were no serious AEs ( $\geq$ grade 3 ).

Conclusions

Mirogabalin may control the progression of CIPN caused by GnP and significantly improved sensory neuropathy. However, since the incidence of AEs is high, mirogabalin should be used with caution.

\section{Introduction}

The treatment of pancreatic cancer (PC) is challenging because most cases are detected at an unresectable stage. Chemotherapy plays a central role in the treatment of PC; regimens include FOLFIRINOX (a four-drug combination of 5-fluorouracil, leucovorin, irinotecan, and oxaliplatin) and gemcitabine plus nab-paclitaxel (nab-PTX) (GnP) ${ }^{1-3}$. However, FOLFIRINOX is unsuitable for elderly patients due to its severe adverse events (AEs), so $\mathrm{GnP}$ therapy is often used as the first-line treatment 4 . AEs such as chemotherapy-induced peripheral neuropathy (CIPN) are frequent and serious problems because they not only reduce quality of life, but also force patients to stop or reduce chemotherapy. Various treatments for CIPN are available, but they are rarely effective ${ }^{5,6}$. Mirogabalin monobenzenesulfonate (Daiichi Sankyo, Tokyo, Japan) is a novel, selective oral $a_{2} \delta$ ligand ${ }^{7}$. It is useful for peripheral neuropathy ${ }^{8,9}$ and may also be effective for CIPN, but studies of its effects on the latter are 
lacking. Therefore, we evaluated the efficacy and safety of mirogabalin for CIPN due to GnP in PC patients.

\section{Method}

\section{Study design}

We conducted a single-center retrospective pilot questionnaire study between January 2019 and August 2020 at Juntendo University Hospital, to evaluate the effects of mirogabalin on CIPN caused by GnP in PC patients. This study was performed in line with the principles of the Declaration of Helsinki. This study was approved by the institutional review board of Juntendo University. All patients provided informed consent.

\section{Patients and setting}

Patients who had previously or were currently receiving GnP for PC, and had taken mirogabalin for at least 2 weeks for numbness, tingling, or pain associated with peripheral neuropathy between January 2019 and June 2020, were included. Participants had to complete a questionnaire to determine the changes in symptoms before and after mirogabalin use. Patients who did not complete the questionnaire due to dementia or refusal were excluded (Figure 1).

The primary study end-point was the change in scores on the patient-reported outcomes version of the Common Terminology Criteria for Adverse Events (PRO-CTCAE) with respect to numbness and tingling. The frequency and severity of numbness and tingling were determined based on items scored from $0-4$, where 0 represents "asymptomatic" and 4 "life-threatening consequences; urgent intervention indicated". Secondary outcomes were the European Organization for Research and Treatment of Cancer (EORTC) Quality of Life Questionnaire-Chemotherapy-Induced Peripheral Neuropathy (QLQ-CIPN20) score and a numerical rating scale (NRS) pain score. The EORTC-QLQ-CIPN20 comprises nine items assessing sensory neuropathy, eight assessing motor function, and three assessing autonomic neuropathy. However, we excluded items 19 and 20. Item 19 is concerned with the difficulty of using pedals and is applicable only to patients who drive a car. Few of our patients drive because of the efficiency of the Tokyo train network. Item 20 pertains to the difficulty of obtaining or maintaining an erection in males; this question was considered inappropriate because few patients undergoing chemotherapy are sexually active. The EORTC-QLQ-CIPN20 items are also scored from 1-4, with 1 representing "not at all" and 4 "very much". The NRS pain scores ranged from 0 (asymptomatic) to 10 (extreme pain).

Mirogabalin is available in 2.5, 5, and $10 \mathrm{mg}$ tablets. We gave patients $5-30 \mathrm{mg} / \mathrm{day}$. The final dosage was based on the individual physician's decision.

\section{Statistical analysis}

We compared variables before and after taking mirogabalin using paired $t$-tests and STATA software (ver. 13.0; StataCorp, College Station, TX, USA). 


\section{Results}

\section{Patient characteristics}

Table 1 summarizes the characteristics of the 25 retrospectively analyzed patients. Their mean age was $66.3 \pm 11.2$ years. Ten patients $(41.7 \%)$ were male. Sixteen patients $(66.7 \%)$ had been diagnosed with diabetes mellitus, of whom ten (41.7\%) had received insulin therapy. The mean hemoglobin A1c (HbA1c) was $6.8 \pm 0.8 \%$, the mean mirogabalin dose was $20.4 \pm 8.2 \mathrm{mg} /$ day and the mean interval between

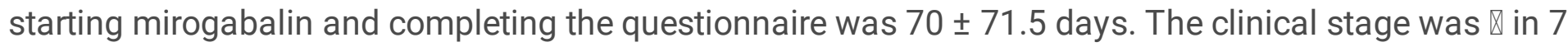

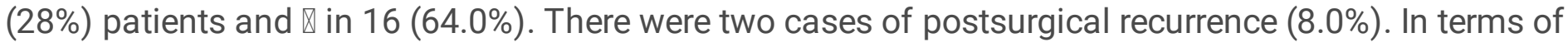
chemotherapy status, twenty-four patients received GnP as first-line therapy, and one as second-line therapy after receiving gemcitabine $+\mathrm{S}-1$. Concomitant with CIPN treatment, vitamin $\mathrm{B}_{12}$ was taken by seven patients, non-steroidal anti-inflammatory drugs or acetaminophen by four, the Chinese herbal medicine goshajinkigan by three, and opioids by three, and changing from pregabalin in one patient.

\section{Efficacy of mirogabalin}

According to the PRO-CTCAE results, no patient experienced significantly increased numbness or tingling severity post-treatment relative to baseline (1.84 $\pm 0.90 \mathrm{vs}$. $1.76 \pm 0.83 ; P=0.63)$, and in no case did numbness and tingling interfere with daily life (1.42 \pm 1.13 vs. $1.46 \pm 1.02 ; P=0.80)$. The mean total EORTC-QLQ-CIPN20 score was $32.6 \pm 11.1$ before taking mirogabalin and $30.6 \pm 9.2$ thereafter $(P=0.12)$. The EORTC-QLQ-CIPN sensory subscale score improved significantly after taking mirogabalin (17.5 \pm 5.9 vs. $15.7 \pm 4.8 ; P=0.02)$, while there were no significant differences in the EORTC-QLQ-CIPN motor (11.7 \pm 4.8 vs. $11.3 \pm 3.9 ; P=0.49)$ or autonomic $(3.4 \pm 1.4$ vs. $3.6 \pm 1.5 ; P=0.31)$ subscale scores. The NRS pain score did not differ significantly before versus after taking mirogabalin $(4.4 \pm 2.0$ vs. $4.1 \pm 2.4 ; P=0.26)$ (Table 2).

Scores for individual items of the sensory and motor neuropathy subscales were also analyzed (Figure 2). The sensory neuropathy-related items showing the largest improvements concerned "tingling in the fingers and hands" and "numbness in the toes and feet", with a mean change of 0.29 . The mean change in "tingling in the toes and feet", "numbness in the fingers and hands", and 'shooting/burning pain in the toes and feet" was 0.25 . The scores for some items pertaining to motor neuropathy improved, while others worsened. The mean change was largest for "cramps in the hands and feet", at 0.21 . The scores for "difficulty holding a pen" and "difficulty climbing stairs" worsened slightly (Figure 3).

\section{Adverse events associated with mirogabalin}

Of the 25 patients, 22 (88.0 \%) experienced AEs of any grade, including somnolence in 20 patients (grade $1, n=17$; grade 2, $n=3$ ), dizziness in 17 (grade $1, n=16$; grade $2, n=1$ ), and edema in three (all grade 1 ). Four patients were unable to continue the medication due to AEs: one each had somnolence and dizziness, somnolence alone, dizziness alone, and difficulty continuing due to edema. The patients who 
experienced AEs felt that the disadvantages of mirogabalin outweighed the advantages, so discontinued its use (Table 3 ).

\section{Discussion}

This is the first study to evaluate the efficacy of mirogabalin for CIPN caused by GnP therapy in PC patients. We found that mirogabalin controlled the progression of CIPN, and sensory neuropathy improved significantly. However, the rate of AEs was relatively high.

Despite the high prevalence of CIPN in association with GnP therapy, there are very few established treatments. It is recommended that, in patients who develop CIPN during chemotherapy, the treatment be delayed or discontinued, or the dose decreased. Effective drugs for CIPN are essential; mirogabalin is one such drug. Nab-PTX is a cytotoxic taxane that frequently causes CIPN. Duloxetine is the only drug recommended by the American Society of Clinical Oncology and Japan Pancreas Society 5,10 for treating established, painful CIPN caused by oxaliplatin or paclitaxel. Randomized controlled trials of duloxetine and pregabalin for taxane-induced CIPN found that pregabalin was more effective ${ }^{11}$. Neuropathy caused by nab-PTX usually presents as sensory neuropathy, manifesting as paresthesias, numbness, and tingling in the toes and fingers. These symptoms are similar to those seen in postherpetic neuralgia and diabetic peripheral neuropathy, and mirogabalin has been shown to be effective for treating them ${ }^{8,9}$. Pregabalin, which has the same mechanism of action as mirogabalin, can improve taxane-induced peripheral neuropathy ${ }^{11}$. We observed no disease progression in CIPN patients after taking mirogabalin compared to baseline, suggesting that it controlled disease progression (which occurs due to cumulative toxicity). Mirogabalin significantly improved sensory neuropathy, including symptoms of CIPN such as tingling and numbness, making it a suitable treatment for these patients.

The CTCAE is generally used to evaluate CIPN, but has several disadvantages. First, the CTCAE was not developed specifically to assess CIPN; moreover, it is not sensitive to changes in disease status and is associated with significant interrater variability ${ }^{12}$. The CTCAE also has a grade range of only $0-4$, which makes it difficult to perform detailed evaluations; the accuracy and precision of the instrument may be inadequate. The PRO-CTCAE was developed to improve the precision of assessment of AEs. The EORTCQLQ-CIPN has a subscale pertaining specifically to symptoms and functional limitations related to CIPN. These instruments provide a more detailed and reliable assessment of CIPN. In addition, quantification of CIPN using the Pain Vision system was also attempted, and correlations with the questionnaire were found ${ }^{13}$.

Adverse effects were more common in this study compared to the literature, where studies have reported somnolence in $8.1-23.9 \%$ of patients receiving mirogabalin, dizziness in $4.9-15.5 \%$, and edema in $2.4-$ $8.5 \% 8,9,14,15$. Most of our patients had somnolence and dizziness. Previous studies on mirogabalinrelated AEs focused on diabetic neuropathy and postherpetic neuralgia, in which somnolence and dizziness are less likely compared to $\mathrm{CIPN}^{8,15}$. CIPN patients tend to receive strong chemotherapy; this increases the likelihood of chemotherapy-related AEs, which may be confused with mirogabalin-related 
ones ${ }^{4,16}$. Studies of AEs associated with mirogabalin reported that the first event tended to occur within 1 month after administration, and that AEs were more frequent in lean women aged over 65 years ${ }^{8,14,15}$. In line with this, we observed somnolence in all of our female patients. The frequency of AEs also increases with the mirogabalin dose. In lean elderly women, who are prone to AEs, a low dose (5 mg/day) during the first month may reduce the likelihood of AEs. Four of our patients discontinued mirogabalin due to grade 1 AEs. The proportion of patients who discontinued mirogabalin was in line with the literature.

Discontinuing or reducing the dosage of mirogabalin is an important option for patients who do not feel that they are deriving any benefits from the medication.

Our study had some limitations. First, it was a single-center pilot study including a small number of cases. Second, only one questionnaire pertained specifically to nab-PTX, which exhibits cumulative toxicity; prospective studies are therefore necessary. Third, the Japanese version of the EORTC-QLQ-CIPN is still being validated (currently in "stage 3 ").

Despite these limitations, this study had several important strengths; it was the to report the effects of mirogabalin on CIPN in PC patients on GnP therapy, and showed that it controlled disease progression. Moreover, using the precise PRO-CTCAE and EORTC-QLQ-CIPN instruments, we demonstrated that mirogabalin is particularly effective for sensory neuropathy.

In conclusion, mirogabalin slowed the progression of CIPN despite cumulative toxicity, and significantly improved sensory neuropathy. However, the rate of AEs was high, so careful dosing and titration are necessary. As this was a pilot study, comparative prospective studies including other drugs are required.

\section{Declarations}

\section{Funding}

The authors received no specific funding for this work.

\section{Author contributions}

All authors contributed to the study conception and design. data collection and analysis were performed by Y.T., T.F., M.U., S.T., W.Y., A.S., K.I., K.O., K.T., S.I. and H.I. The first draft of the manuscript was written by Y.T. and all authors commented on previous versions of the manuscript. All authors read and approved the final manuscript.

\section{Conflicts of Interest}

The authors have no financial or proprietary interests in any material discussed in this article.

\section{References}


1 Tempero, M. A. NCCN Guidelines Updates: Pancreatic Cancer. J. Natl. Compr. Canc. Netw. 17, 603605, doi:10.6004/jnccn.2019.5007 (2019).

2 Sohal, D. P. S. et al. Metastatic Pancreatic Cancer: ASCO Clinical Practice Guideline Update. J. Clin. Oncol. 36, 2545-2556, doi:10.1200/JC0.2018.78.9636 (2018).

3 Ducreux, M. et al. Cancer of the pancreas: ESMO Clinical Practice Guidelines for diagnosis, treatment and follow-up. Ann. Oncol. 26 Suppl 5, v56-68, doi:10.1093/annonc/mdv295 (2015).

4 Von Hoff, D. D. et al. Increased survival in pancreatic cancer with nab-paclitaxel plus gemcitabine. N. Engl. J. Med. 369, 1691-1703, doi:10.1056/NEJMoa1304369 (2013).

5 Loprinzi, C. L. et al. Prevention and Management of Chemotherapy-Induced Peripheral Neuropathy in Survivors of Adult Cancers: ASCO Guideline Update. J. Clin. Oncol. 38, 3325-3348, doi:10.1200/JC0.20.01399 (2020).

6 Dorsey, S. G. et al. The National Cancer Institute Clinical Trials Planning Meeting for Prevention and Treatment of Chemotherapy-Induced Peripheral Neuropathy. J. Natl. Cancer Inst. 111, 531-537, doi:10.1093/jnci/djz011 (2019).

7 Domon, Y. et al. Binding Characteristics and Analgesic Effects of Mirogabalin, a Novel Ligand for the alpha2delta Subunit of Voltage-Gated Calcium Channels. J Pharmacol Exp Ther 365, 573-582, doi:10.1124/jpet.117.247551 (2018).

8 Kato, J. et al. Mirogabalin for the management of postherpetic neuralgia: a randomized, doubleblind, placebo-controlled phase 3 study in Asian patients. Pain 160, 1175-1185, doi:10.1097/j.pain.0000000000001501 (2019).

9 Baba, M., Matsui, N., Kuroha, M., Wasaki, Y. \& Ohwada, S. Mirogabalin for the treatment of diabetic peripheral neuropathic pain: A randomized, double-blind, placebo-controlled phase III study in Asian patients. J Diabetes Investig 10, 1299-1306, doi:10.1111/jdi.13013 (2019).

10 Okusaka, T. et al. Clinical Practice Guidelines for Pancreatic Cancer 2019 From the Japan Pancreas Society: A Synopsis. Pancreas 49, 326-335, doi:10.1097/MPA.0000000000001513 (2020).

11 Salehifar, E. et al. Comparison of the Efficacy and Safety of Pregabalin and Duloxetine in TaxaneInduced Sensory Neuropathy: A Randomized Controlled Trial. Clin. Drug Investig. 40, 249-257, doi:10.1007/s40261-019-00882-6 (2020).

12 Cavaletti, G. et al. Chemotherapy-Induced Peripheral Neurotoxicity assessment: a critical revision of the currently available tools. Eur. J. Cancer 46, 479-494, doi:10.1016/j.ejca.2009.12.008 (2010).

13 Yoshida, Y. et al. The Relationship Between Evaluation Methods for Chemotherapy-Induced Peripheral Neuropathy. Sci. Rep. 9, 20361, doi:10.1038/s41598-019-56969-9 (2019). 
14 Kato, J. et al. Safety and Efficacy of Mirogabalin for Peripheral Neuropathic Pain: Pooled Analysis of Two Pivotal Phase III Studies. Clin. Ther., doi:10.1016/j.clinthera.2021.03.015 (2021).

15 Inage, K. et al. Frequency of Adverse Drug Reactions and Analgesic Effects of Mirogabalin during Treatment of Peripheral Neuropathic Pain: A Retrospective Clinical Investigation. Spine Surg Relat Res 4, 354-357, doi:10.22603/ssrr.2020-0042 (2020).

16 Ueno, H. et al. Phase I/II study of nab-paclitaxel plus gemcitabine for chemotherapy-naive Japanese patients with metastatic pancreatic cancer. Cancer Chemother. Pharmacol. 77, 595-603, doi:10.1007/s00280-016-2972-3 (2016).

\section{Tables}

Table 1. Patient characteristics 


\begin{tabular}{|c|c|}
\hline & $n=25$ \\
\hline Age, y.o. & $65.9 \pm 11.2$ \\
\hline \multicolumn{2}{|l|}{ sex } \\
\hline Male, $(\%)$ & $10(40.0)$ \\
\hline Female, (\%) & $15(60.0)$ \\
\hline Diabetes mellitus, (\%) & $16(64.0)$ \\
\hline Insulin user, (\%) & $10(40.0)$ \\
\hline Metformin, (\%) & $2(8)$ \\
\hline $\mathrm{HbA} 1 \mathrm{c}, \%$ & $6.8 \pm 0.8$ \\
\hline Mean mirogabalin dose, mg/day & $20.4 \pm 8.2$ \\
\hline Mean period to the time of the questionnaire, day & $70 \pm 71.5$ \\
\hline \multicolumn{2}{|l|}{ Clinical Stage } \\
\hline$\otimes(\%)$ & $7(28.0)$ \\
\hline$\otimes(\%)$ & $16(64.0)$ \\
\hline Postsurgical recurrence & $2(8.0)$ \\
\hline \multicolumn{2}{|l|}{ Chemotherapy status } \\
\hline GnP therapy $1 \mathrm{st} / 2 \mathrm{nd}$ line & $24 / 1$ \\
\hline Median nab-PTX, times & $6(5-10)$ \\
\hline Median nab-PTX, mg & $1191(890-1904)$ \\
\hline \multicolumn{2}{|l|}{ Concomitant anti CIPN drug } \\
\hline None, $(\%)$ & $11(44)$ \\
\hline Switch from Pregabalin, (\%) & $1(4.0)$ \\
\hline Vit.B12, (\%) & $7(28.0)$ \\
\hline Goshajinkigan, (\%) & $3(12.0)$ \\
\hline NSAIDs or Acetaminophen, (\%) & $4(16.0)$ \\
\hline Opioids, (\%) & $3(12.0)$ \\
\hline
\end{tabular}

Note. HbA1c: Hemoglobin A1c, GnP: Gemcitabine/nab-paclitaxel, nab-PTX: nab-paclitaxel, CIPN: chemotherapy-induced peripheral neuropathy, NSAIDs: non-steroidal anti-inflammatory drugs. 


\begin{tabular}{|llll|}
\hline \multicolumn{1}{|l}{ Pre } & Post & P value \\
\hline PRO-CTCAE, mean & & & \\
\hline Severity & $1.84 \pm 0.90$ & $1.76 \pm 0.83$ & 0.63 \\
\hline Interference & $1.42 \pm 1.13$ & $1.44 \pm 1.02$ & 0.80 \\
\hline EORTC-QLQ-CIPN20, mean & & & \\
\hline Total scale & $32.6 \pm 11.1$ & $30.6 \pm 9.2$ & 0.12 \\
\hline Sensory subscale & $17.5 \pm 5.9$ & $15.7 \pm 4.8$ & $\mathbf{0 . 0 2}$ \\
\hline Motor subscale & $11.7 \pm 4.8$ & $11.3 \pm 3.9$ & 0.49 \\
\hline Autonomic subscale & $3.4 \pm 1.4$ & $3.6 \pm 1.5$ & 0.31 \\
\hline NRS of neuropathy & $4.4 \pm 2.0$ & $4.1 \pm 2.4$ & 0.26 \\
\hline
\end{tabular}

Note. CIPN: chemotherapy-induced peripheral neuropathy, GnP: Gemcitabine/nab-paclitaxel, PC: pancreatic cancer, PRO-CTCAE: patients reported outcome Common Terminology Criteria for Adverse Events, EORTC: European Organization for Research and Treatment of Cancer, NRS: numerical rating scale.

Table.3 Safty of mirogabalin for CIPN patients.

\begin{tabular}{|lllll|}
\hline AEs & Any grade (\%) & Grade 1 & Grade 2 & $\geq$ Grade3 \\
\hline Somnolence & $20(80)$ & 17 & 3 & 0 \\
\hline Dizziness & $17(68)$ & 16 & 1 & 0 \\
\hline Edema & $3(12)$ & 3 & 0 & 0 \\
\hline Discontinuation of mirogabalin due to AEs & $4(16)$ & - & - & - \\
\hline
\end{tabular}

Note: CIPN: chemotherapy-induced peripheral neuropathy, AEs: adverse events

\section{Figures}


Patients with pancreatic cancer who have received or are currently receiving $\mathrm{GnP}$

Patients with CIPN who have received or are receiving treatment with mirogabalin $(\mathrm{n}=26)$

Excluded $(\mathrm{n}=1)$

№t consent $(\mathrm{n}=1)$

Dementia $(\mathrm{n}=0)$

ㅁ Not continue to take mirogabalin for 2 weeks $(\mathrm{n}=0)$

Evaluate endpoint

$(\mathrm{n}=25)$

\section{Figure 1}

Study flowchart. GnP, Gemcitabine/nab-paclitaxel therapy; CIPN, chemotherapy-induced peripheral neuropathy

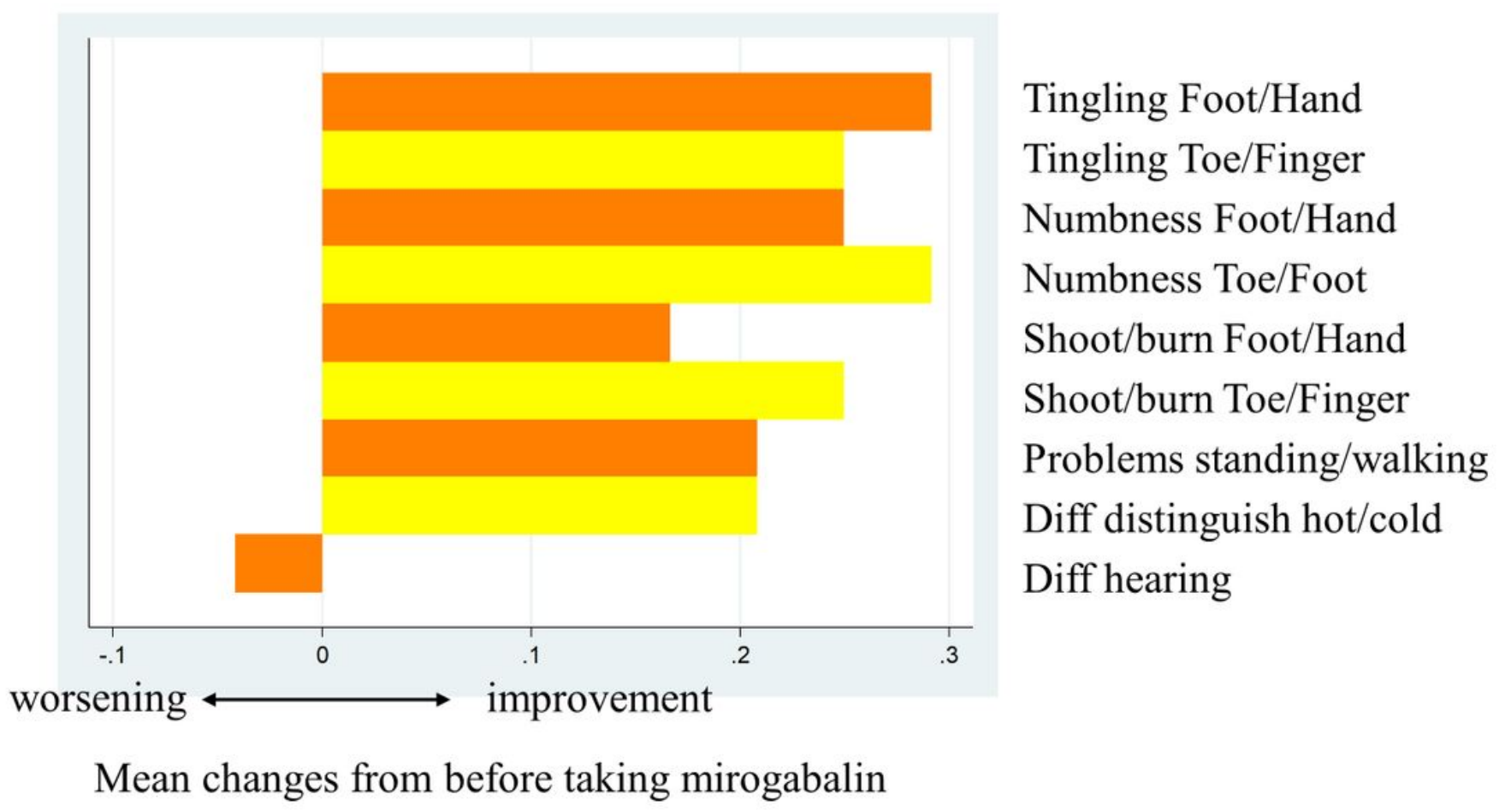


Figure 2

Mean changes in sensory neuropathy subscale scores before and after taking mirogabalin.

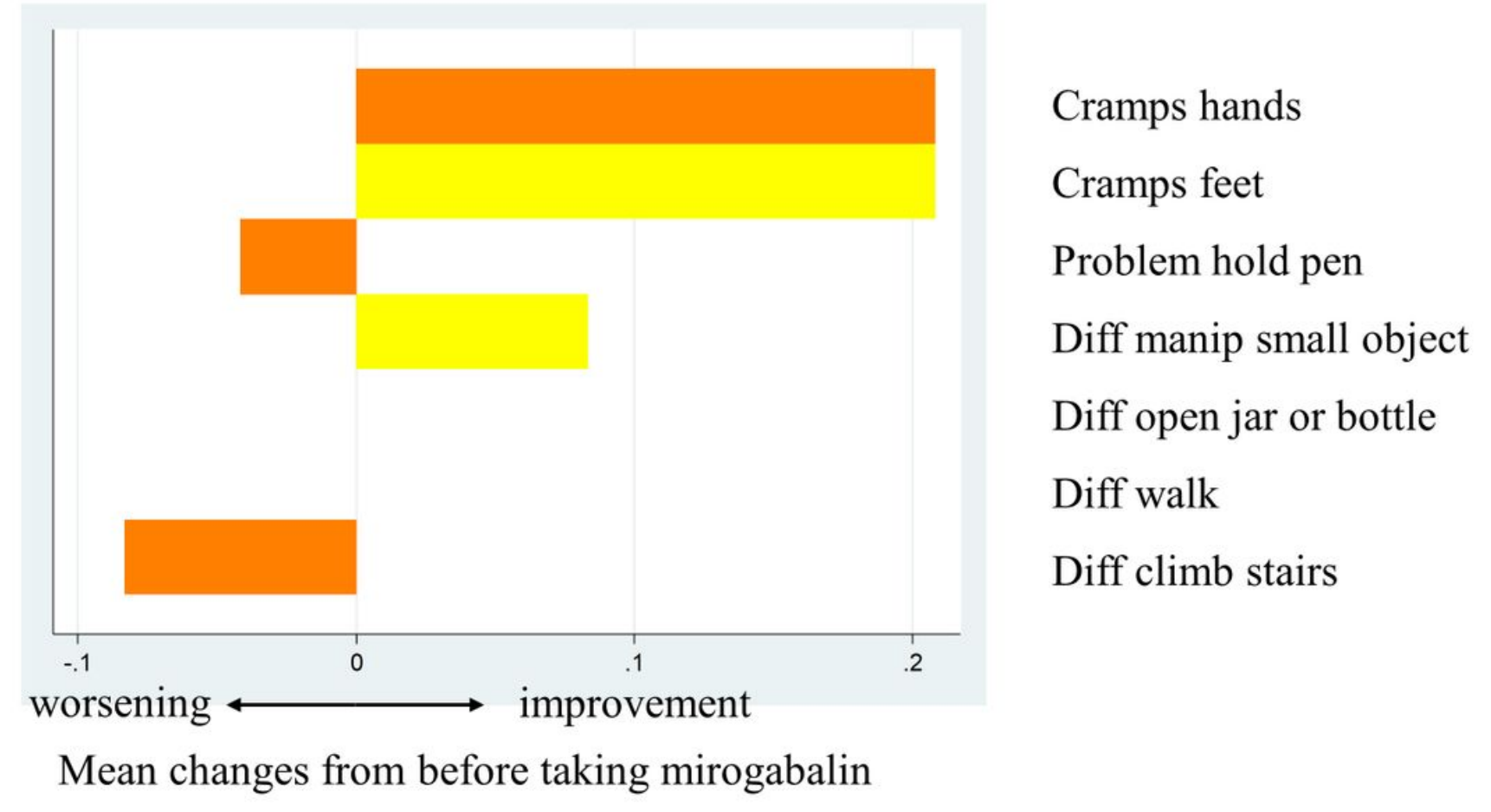

\section{Figure 3}

Mean changes in motor neuropathy subscale scores before and after taking mirogabalin. 